\title{
Primary Isolated Extra Nodal Esophageal Hodgkin Lymphoma in Diabetic Patient
}

\author{
Mayson A. Alameen ${ }^{*}$, Ahmed Ibrahim ${ }^{2}$, Touqeer Ahmed Siddiqui ${ }^{3}$, Mervat Mohrous ${ }^{3}$, \\ Fadhel Alotaibi3 ${ }^{3}$ Abdel Aziz Al Hamad3, Hussein Farghaly3 \\ ${ }^{1}$ Department of Oncology, Prince Sultan Medical City, Riyadh, Saudi Arabia \\ ${ }^{2}$ Researches \& Biostatistics Department, Prince Sultan Cardiac Center, Al Hassa, Saudi Arabia \\ ${ }^{3}$ Histopathology, Prince Sultan Medical City, Riyadh, Saudi Arabia \\ ${ }^{4}$ Nuclear Medicine Division, Prince Sultan Medical City, Riyadh, Saudi Arabia \\ Email: abrahimsonsen@gmail.com, tauqeer96@gmail.com, mervat_mahrous@yahoo.com, fadelotb@gmail.com, \\ aalhamad@psmmc.mes.sa, hussen2h@yahoo.com, ^masoona.alameen@gmail.com
}

How to cite this paper: Alameen, M.A., Ibrahim, A., Siddiqui, T.A., Mohrous, M., Alotaibi, F., Al Hamad, A.A. and Farghaly, H. (2021) Primary Isolated Extra Nodal Esophageal Hodgkin Lymphoma in Diabetic Patient. Journal of Cancer Therapy, 12, 497504

https://doi.org/10.4236/jct.2021.129043

Received: June 21, 2021

Accepted: September 5, 2021

Published: September 8, 2021

Copyright $\odot 2021$ by author(s) and Scientific Research Publishing Inc. This work is licensed under the Creative Commons Attribution International License (CC BY 4.0).

http://creativecommons.org/licenses/by/4.0/

\begin{abstract}
Primary isolated esophageal Hodgkin's lymphoma is seldom in localization and when seen, it is usually the non-Hodgkin's type. We reported a 68-year-old, diabetic and ex-smoker, presented to our hospital complaining of progressive difficulty of swallowing for nine months for both solid and liquid. He was on proton pump inhibitors. Recently, he had fever and drenching night sweat and significant loss of weight. He was diagnosed as a case of isolated esophageal Hodgkin's lymphoma that was diagnosed based on endoscopic and radiological findings. Clinical manifestations, radiological imagines and histopathological features were described. Clinical management, fellow-up and outcome were precisely discussed.
\end{abstract}

\section{Keywords}

Esophagus, Chemotherapy, Radiotherapy, Non-Hodgkin's Lymphoma

\section{Introduction}

The esophagus is an unusual site for lymphomas less than $1 \%$ of patients with lymphoma and is commonly seen secondary to mediastinal nodes or gastric lymphoma [1] [2]. Isolated primary esophageal lymphoma is extremely rare, and when it is diagnosed, it is always non-Hodgkin. However, only, less than twenty percent of lymphomas present with extranodal localization as known [3]. Hodgkin's lymphoma (HL) is typically a systemic disease characterized by the presence of Reed-Sternberg cells as considered to be a type of B cells. These cells 
however only occupy a very small proportion $(<5 \%)$ of the overall cell population of the affected lymph node [4].

\section{Case Presentation}

This is a case report of 68 years old diabetic man on oral hypoglycemic agent, he had mild hearing impairment due to otosclerosis presented to gastroenterology team clinic in our hospital with progressive difficulty of swallowing for both solid and liquid for nine month he had history of gastroesphgeal reflux disease for long time has been on Pantoprazole switched later on to Esomprazole. Recently he had fever and drenching night sweat and significant loss of weight around 20 kilogram, patient had positive family history of malignancy in his eldest sister but not of any specific nature. He has been smoker for 30 years or more, patient has history of esophagitis for one year. His physical examination revealed pale not jaundice, no palpable masses or enlarged lymph node and no organomegaly. Investigations plan was to perform endoscopy with fine needle aspiration (FNA). Informed consent was obtained from the patient after explaining the benefits and risks of the procedure.

\section{Diagnosis}

Endoscopic ultrasound (EUS) with fine needle aspiration (FNA) were done under full sterilization procedure, the patient was connected to the monitoring devices and placed in the left lateral position. Continuous oxygen was provided with a nasal cannula and IV medicine administered through an indwelling cannula. After adequate conscious sedation was achieved, the patient was intubated and the scope advanced under direct visualization to the second part of duodenum.

The second part of duodenum was identified by visual landmarks. The scope was subsequently removed slowly while carefully examining the color, texture, anatomy, and integrity of the mucosa on the way out. The patient was subsequently transferred to the recovery area in satisfactory condition.

The procedure was successfully done without any complication and Nexium $40 \mathrm{mg}$ was prescribed to the patient. The patient was discharged to home with close outpatient follow-up Figure 1. Then the patient returned to gastroenterology clinic after two weeks, histopathology examination of the biopsies of the lower esophagus mass show fragments of gastroesophageal junction mucosa infiltrated by atypical large cells (Figure 2(A) \& Figure 2(B)) in a background of mixed inflammatory cells including eosinophils. The large cells have monolobed, and polylobed large nuclei with vesicular chromatin and large eosinophilic central nucleoli. Reed-Sternberg cells with classic "owl eye" appearance are also present. The neoplastic cells are positive for CD30 and PAX5 (dim), and MUM-1 immunostains (Figures 3(C)-(E)). They are negative for CD45, CD3, CD20, and Alk-1. EBV in situ hybridization (EBER) is positive (Figure 3(E)). These features are consistent with classical Hodgkin lymphoma. 


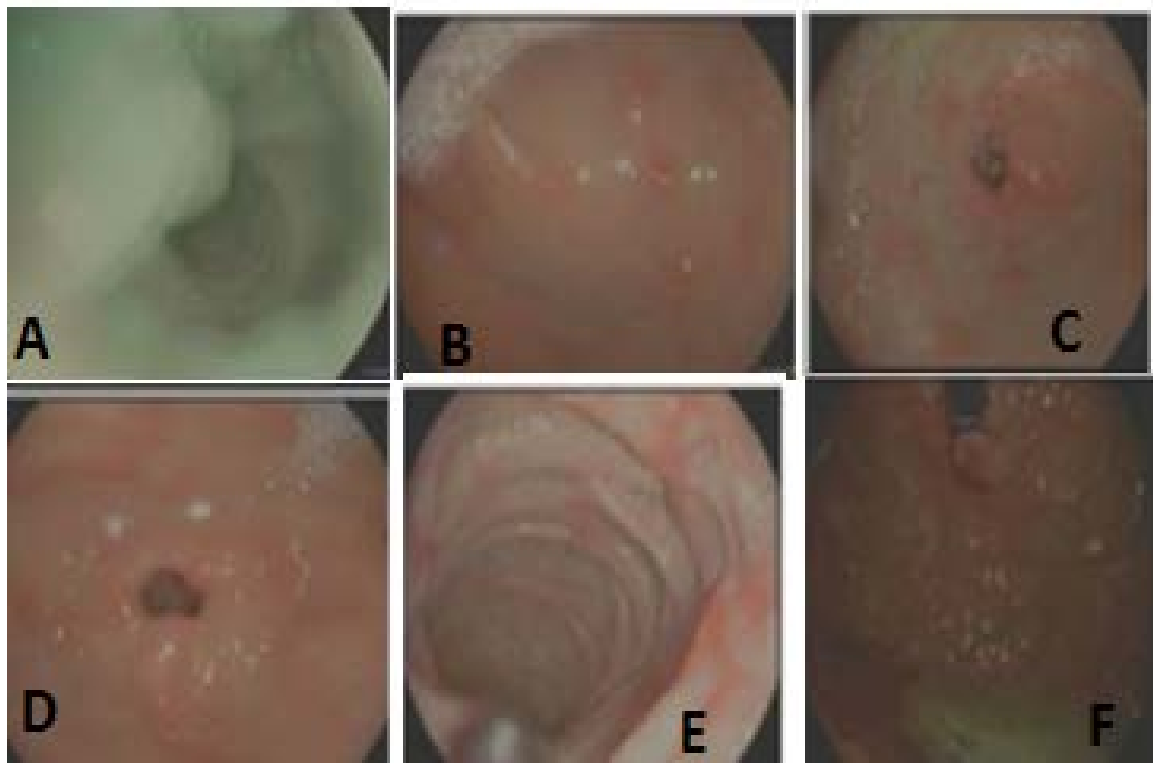

Figure 1. Esophago-gastroduodenoscopy. (A) Prominent fold at the lower esophageal area from $35 \mathrm{~cm}$ to $40 \mathrm{~cm}$. (B) ulcer around $1 \mathrm{~cm}$ just above the gastroesophageal junction (GEJ). (C), (D), (E) diffuse erythema and erosions were observed all over the stomach and at duodenum. (F) Acardiac prominent bulge was seen just below the GEJ. Multiple biopsies were obtained from the observed lesions for pathological evaluation.

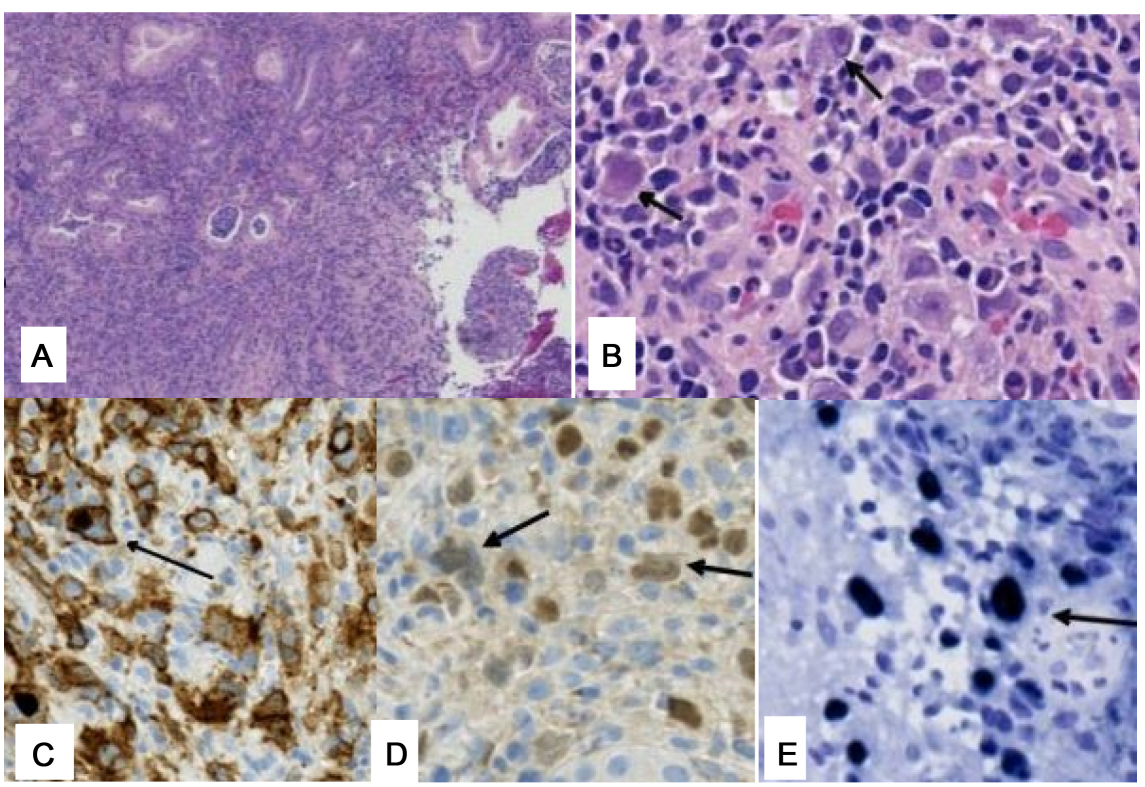

Figure 2. Histopathology and \& Immunochemistry (as above). Describe (A), (B), (C), (E) (as in Figure 1). (A), (B) Mixed inflammatory cells including eosinophils. The large cells Reed-Sternberg cells with classic "owl eye" appearance present. positive for CD30 and PAX5 (dim), and MUM-1 immunostains (C), (D)-(E). They are negative for CD45, CD3, CD20, and Alk-1. EBV in situ.

The patient was referred immediately to oncology specialty for further treatment history and proper examination then requested tumor markers a complete base line and staging work up initiated by X-ray shows an abnormal. 


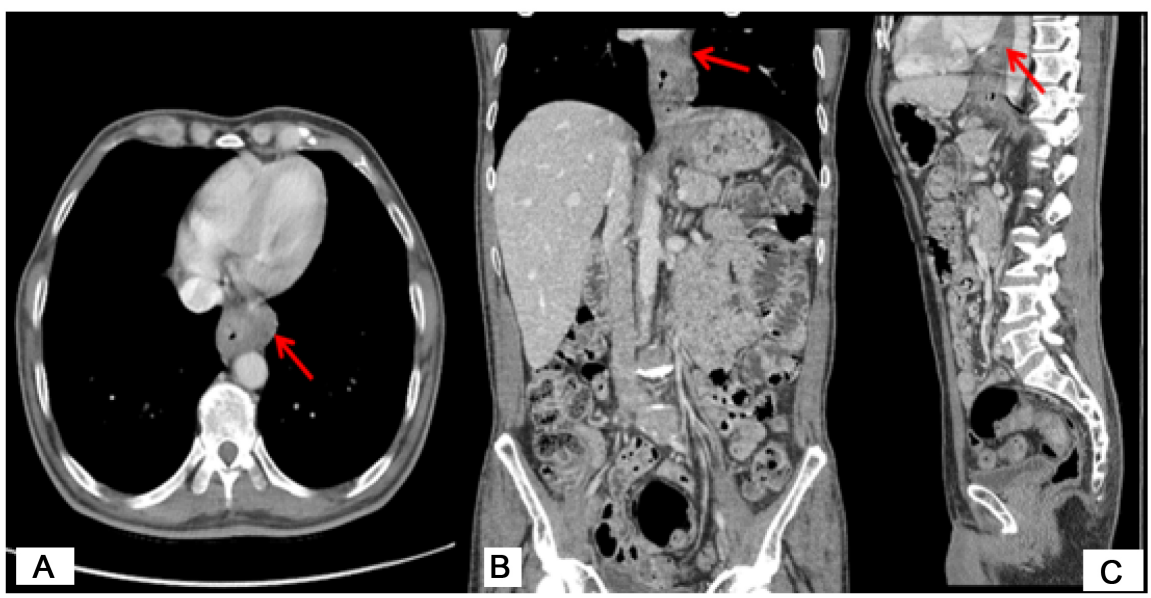

Figure 3. Radiology findings contrast enhanced CT-Chest, abdomen and pelvis. (A): enlarged lymph nodes in the perioseophageal region and small hiatus hernia, $(B)$ and $(C)$ : diffuse circumferential wall thickening involving almost the whole length of the esophagus.

Contrast enhanced CT for chest, abdomen and pelvis: There is diffuse circumferential wall thickening involving almost the whole length of the esophagus with small hiatus hernia, however, at the lower part of the esophagus above the hiatus hernia, there is a more pronounced circumferential soft tissue thickening (red arrow) merging with the rest of the diffuse esophageal wall thickening. There are multiple enlarged lymph nodes in the perioseophageal region and gastrohepatic ligament, largest measuring about $1 \mathrm{~cm}$ in short axis diameter Figure 4.

\section{Case Management}

The patient was diagnosed as a case Hodgkin lymphoma for chemotherapy. The case was discussed in the oncology board and treatment protocol was approved. The patient received 4 cycles of combination chemotherapy of doxorubicin 36 $\mathrm{mg}$, bleomycin $14.4 \mathrm{mg}$, vinblastine $8 \mathrm{mg}$ and DICT $540 \mathrm{mg}$ with dexamethasone $8 \mathrm{mg}$ and hydrocortisone $100 \mathrm{mg}$ as full dose adjusted according to the surface area 1.44. Post chemotherapy medication given as well included Granulocyte-colony stimulating factor (G-CSF) after 24 hours antiemetic (granisetron, metoclopramide, ranitidine) and antibiotics (ciprofloxacin, bactrim) were administered. Overall, he tolerated the chemotherapy regimen well, after two cycles he was re-evaluated by PET Scan re-which showed partial improvement compared to baseline findings. Figure 4: it showed in Figure 4 (A) the baseline findings there was fused axial, coronal and sagittal PET/CT and MIP image showed high FDG avid pronounced circumferential thickening at lower esophageal third just superior to a hiatus hernia (arrow) with SUV max of 9.4 with no other FDG avid lesion in the rest of the scanned body. (B) Interim after 4cycles of chemotherapy fused axial, coronal and sagittal PET/CT and MIP image showed significant morphological and metabolic regression of the previously seen distal 


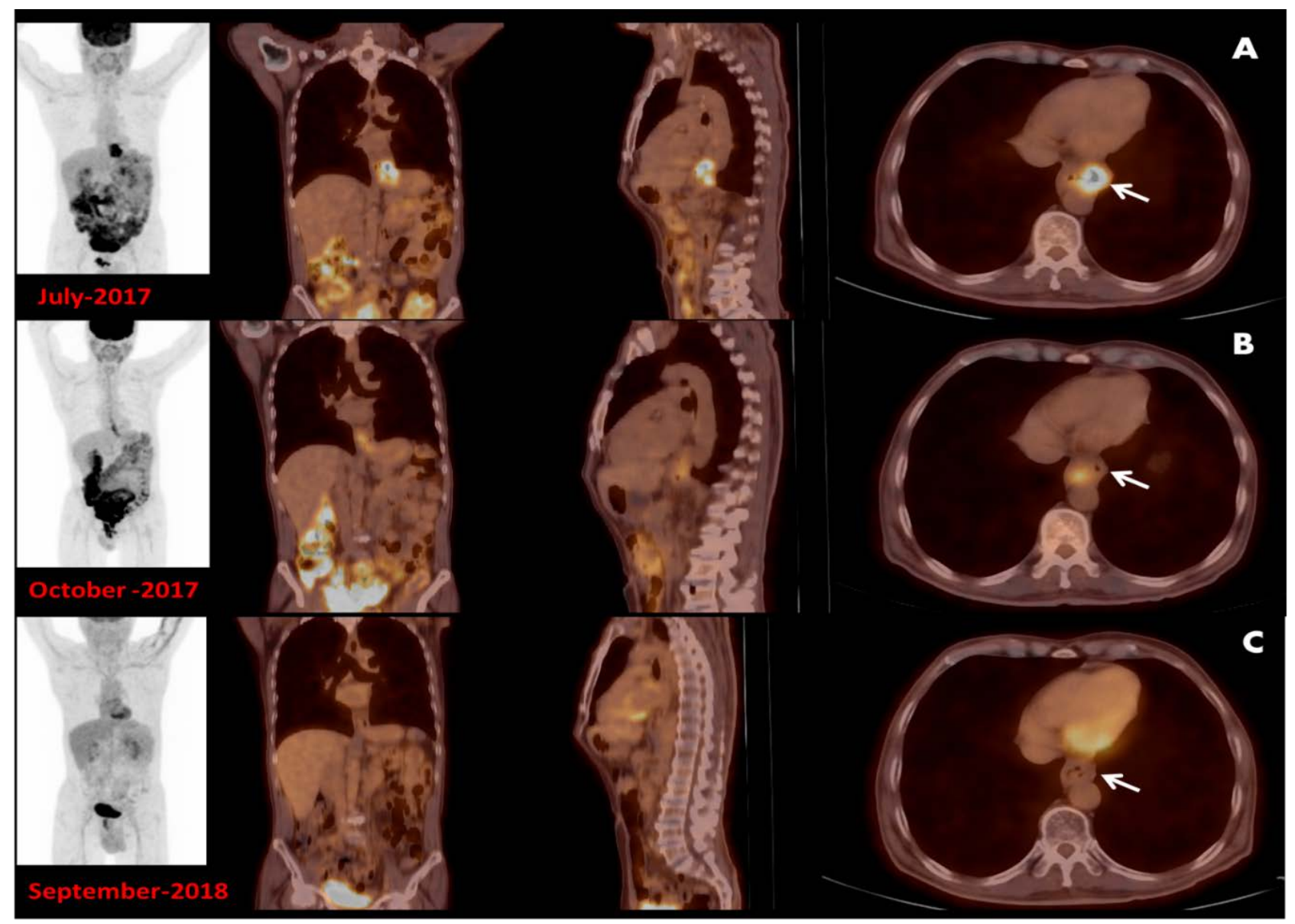

Figure 4. FDG PET/CT. (A) Thickening at lower esophageal third superior to a hiatus hernia. (B) Significant morphological and metabolic regression of the previously seen distal esophageal lymphomatous lesion. (C) Post treatment image showed no residual metabolic activity seen at the previous distal esophageal lymphomatous lesion and no FDG avid lesion.

esophageal lymphomatous lesion with interval decrease in SUV max to 4.6 (Deauville score 4) with no other FDG avid lesion in the rest of the scanned body indicating partial response to the given chemotherapy associated with diffuse esophageal FDG uptake likely due to associated esophagitis. (C) Post treatment fused axial, coronal and sagittal PET/CT and MIP image showed no residual metabolic activity could be seen in the region of the previously seen distal esophageal lymphomatous lesion and no FDG avid lesion in the scanned body (Deauville score 1) indicating complete metabolic response to the given treatment.

\section{Discussion}

We present a unique case of Isolated Extra nodal Esophageal hodgkin lymphoma in elderly gentleman one fifth of lymphomas present with extranodal localization [4]. The Diagnostic workup in Hodgkin Lymphoma based on ESMO 2020 Clinical Practice Guidelines for diagnosis, treatment and follow-up [5]. With standardized protocol implementation, overall long-term survival rate is less than 80 , still there are approximately $30 \%$ of patients who relapse after first line 
therapy [6].

There is paucity in published literature on isolated esophageal Hodgkin's lymphoma. Most cases of existed primary esophageal Hodgkin's disease that were previously reported had concomitant peripheral lymphadenopathy at the time of lesion detection, arose secondarily by extension from involved adjacent lymph nodes or by spread from the gastric fundus, or recurred after a previously treated Hodgkin's disease. On the underpinning of these findings, true primary Hodgkin's lymphoma of the esophagus is extraordinary being reported in less than four cases worldwide [7] [8] [9]. Lymphoma of the esophagus occurs more often in the distal esophagus [10]. Nonetheless, Hodgkin's disease involves predominantly the upper or mid esophagus [11]. As with lymphoma elsewhere in the gastrointestinal tract, the radiographic findings of esophageal lymphoma are somewhat nonspecific and have been reported as multiple submucosal nodules sometimes appearing as diffuse fine modularity [12] or as enlarged varicoid tortuous longitudinal folds, a single large intramural mass, polypoid masses with or without ulceration, an achalasia like tapered narrowing of the distal esophagus, and irregular and sometimes infiltrating strictures indistinguishable from esophageal carcinoma [10] [12].

This case highlights the difficulty of diagnosing isolated primary esophageal lymphoma, even when symptomatic. Because primary lymphoma arises typically in the submucosal or lamina propria lymphoid patches of the gut wall, findings of routine endoscopic biopsies, which sample the mucosa, are often normal or show nonspecific inflammatory changes [13]. Even deep biopsies with rigid endoscopy are sometimes no diagnostic because lymphomatous changes in the esophageal wall may be patchy and missed in spite of the concern caused by the radiologic and endoscopic appearances [14].

Experts recommended the significance of early diagnosis on response to local or systemic treatment is usually of good outcome [15]. Hence, oncologists and radiologists should be aware of this disease. Even though the disease is rare, primary esophageal Hodgkin's lymphoma has to be included in the differential diagnosis of atypical esophageal lesions, even in asymptomatic patients.

\section{Conclusion}

Esophageal Hodgkin lymphoma is uncommonly to be isolated and its location, Hodgkin lymphoma is seldom diagnosed in the geriatric population. This case illustrates rapid growth and significant morbidity, after treating no relapses and unexpected recurrence.

\section{Acknowledgements}

We would like to thank our outstanding radiologist, all the nurses, technicians and physicians who participated in management of this case.

\section{Conflicts of Interest}

The authors declare no conflicts of interest regarding the publication of this paper. 


\section{Learned Lessons}

- Early diagnosis on improving response to local or systemic treatment and having a good outcome. Hodgkin lymphoma should be included in differential diagnosis of atypical esophageal lesions, even on asymptomatic patients.

- Oncologists and radiologists should be aware of uncommon presentations of this disease.

\section{Ethical Approval}

The research was approved by Research and Bioethics Committee at Prince Sultan Medical City according to good clinical practice guidelines. Written informed consent was obtained from the patient for publication of his case report and accompanying image.

\section{Funding}

There was no fund to this research.

\section{References}

[1] Oğuzkurt, L., Karabulut, N., Cakmakci, E. and Besim, A. (1997) Primary NonHodgkin's Lymphoma of the Esophagus. Abdominal Radiology, 22, 8-10. https://doi.org/10.1007/s002619900129

[2] Jones, A.S., Roland, N.J., Hamilton, J., Rowley, H. and Nandapalan, V. (1996) Malignant Tumours of the Cervical Oesophagus. Clinical Otolaryngology and Allied Sciences, 21, 49-53. https://doi.org/10.1111/j.1365-2273.1996.tb01024.x

[3] Freeman, C., Berg, J.W. and Cutler, S.J. (1972) Occurrence and Prognosis of Extranodal Lymphomas. Cancer, 29, 252-260. https://doi.org/10.1002/1097-0142(197201)29:1<252::AID-CNCR2820290138>3.0.C $\underline{\mathrm{O} ; 2-\#}$

[4] Kalogeropoulos, I.V., Chalazonitis, A.N., Tsolaki, S., et al. (2009) A Case of Primary Isolated Non-Hodgkin's Lymphoma of the Esophagus in an Immunocompetent $\mathrm{Pa}$ tient. World Journal of Gastroenterology, 15, 1901-1903. https://doi.org/10.3748/wig.15.1901

[5] Lordick, F., Mariette, C., Haustermans, K., Obermannová, R. and Arnold, D. (2016) Oesophageal Cancer: ESMO Clinical Practice Guidelines for Diagnosis, Treatment and Follow-Up. Annals of Oncology, 27, v50-v57. https://doi.org/10.1093/annonc/mdw329

[6] Witkowska, M., Majchrzak, A. and Smolewski, P. (2015) The Role of Radiotherapy in Hodgkin's Lymphoma: What Has Been Achieved during the Last 50 Years? BioMed Research International, 2015, Article ID: 485071. https://doi.org/10.1155/2015/485071

[7] Rosenberg, S.A., Diamond, H.D., Jaslowitz, B. and Craver, L.F. (1961) Lymphosarcoma: A Review of 1269 Cases. Medicine, 40, 31-84. https://doi.org/10.1097/00005792-196102000-00002

[8] Loeb, D.S., Ribeiro, A. and Menke, D.M. (1999) Hodgkin's Disease of the Esophagus: Report of a Case. The American Journal of Gastroenterology, 94, 520-522. https://doi.org/10.1111/j.1572-0241.1999.00802.x

[9] Stein, H.A., Murray, D. and Warner, H.A. (1981) Primary Hodgkin's Disease of the 
Esophagus. Digestive Diseases and Sciences, 26, 457-461.

https://doi.org/10.1007/BF01313591

[10] Carnovale, R.L., Goldstein, H.M., Zornoza, J. and Dodd, G.D. (1977) Radiologic Manifestations of Esophageal Lymphoma. $A J R, 128,751-754$.

https://doi.org/10.2214/ajr.128.5.751

[11] Rosenberg, S.A., Diamond, H.D., Jaslowitz, B. and Craver, L.F. (1961) Lymphosarcoma: A Review of 1269 Cases. Medicine, 40, 31-84. https://doi.org/10.1097/00005792-196102000-00002

[12] Gelb, A.B., Medeiros, L.J., Chen, Y.-Y., Weiss, L.M. and Weidner, N. (1997) Hodgkin's Disease of the Esophagus. American Journal of Clinical Pathology, 108, 593-598. https://doi.org/10.1093/ajcp/108.5.593

[13] Levine, M.S., Sunshine, A.G., Reynolds, J.C. and Saul, S.H. (1985) Diffuse Nodularity in Esophageal Lymphoma. $A J R, 145,1218-1220$. https://doi.org/10.2214/ajr.145.6.1218

[14] Carbone, P.P., Kaplan, H.S., Musshoff, K., Smithers, D.W. and Tubiana, M. (1971) Report of the Committee on Hodgkin's Disease Staging Classification. Cancer Research, 31, 1860-1861.

[15] Taal, B.G., Van Heerde, P. and Somers, R. (1993) Isolated Primary Esophageal Involvement by Lymphoma: A Rare Cause of Dysphagia-Two Case Histories and a Review of Other Published Data. Gut, 34, 994-998.

https://doi.org/10.1136/gut.34.7.994 